\title{
The short-term assessment, rehabilitation, reablement service "starrs": a rapid response team to support the frail older person to remain at home
}

\begin{abstract}
This paper describes the Rapid Response Team (STARRS)in Greater London. It comprises a large data set with over 17,000 patients over a 4 year period from February 2016 to February 2020. The intermediate care team is able to support the older person at home in clinical crises or in need of social support thus avoiding hospital admissions; this is done through daily visits and geriatricians review via daily virtual ward rounds. Hospital admission avoidance (HAA) and re-admission rate at 30 days (HRA30) has been in excess of $80 \%$ and less than $10 \%$ respectively. Clinical governance was shared between geriatricians and patients 'General Practitioner. The service is popular with General Practitioners and with patients. Economic evaluation reveals the service may also be cost effective with significant potential savings.
\end{abstract}

Keywords: rapid response team, hospital avoidance, hospital readmissions, cost analysis
Volume 5 Issue 3 - 2020

\author{
E Chua,' LPThum,' N Shah,' E Swart,' V \\ Kolli,' M Mistry,' MC Patel, ${ }^{2}$ P Chua ${ }^{3}$ \\ 'Northwick Park Hospital, London North-West University \\ HealthCare NHS Trust, UK \\ ${ }^{2}$ Brent Clinical Commissioning Group, UK \\ ${ }^{3}$ University of Manchester, UK
}

Correspondence: E Chua, Northwick Park Hospital, Watford Road, Harrow, Middlesex, HAI 3UJ, UK,

Email Eddy.cha@nhs.net

Received: May 26, 2020 | Published: June 08, 2020
Abbreviations: A\&E, accident and emergency; CCG, clinical commissioning group; GP, general practitioners; LAS, london ambulance service; HAA, hospital admission avoidance, HRA30 days, hospital avoidance at 30 days; KPI, key performance status; RRT, rapid response team; STARRS, short-term assessment, rehabilitation, reablement service

\section{Background}

The Rapid Response Service (RRS) called STARRS (Short Term Assessment, Reablement, Rehabilitation Service) serves the Borough of Brent, in North-West London with a population of 330,795 of which, 39,995 are over the age of 65 and above. ${ }^{1}$ The Borough of Brent is served by a single clinical commissioning group and also one social service team. STARRS was set up in late2010. It was initially nurse led and had geriatrician support onan ad hoc basics.

From mid 2013, there was regular input from a geriatrician three sessions a week. In 2014, there were daily virtual ward rounds and also geriatricians support until 10pm, 7 days a week. The service was designed to help support GP's to manage clinical and social crisis at home. STARRS also accepts referrals from London Ambulance Service (LAS) after a face to face assessment and from A\&E up to 48 hours post presentation.

There is limited data in the literature for such a service. We provide a large data set for such a service, with hospital admissions whilst under the service, hospital admissions at 30 days post discharge and a cost analysis.

\section{Method}

\section{The rapid response team (RRT)}

It is a multi-disciplinary team of nurses, therapists (physiotherapists and occupational therapists), paramedics, dietitian and geriatricians. Patients remain in their home but are visited daily by nurses and/or therapists. They are then discussed in daily virtual ward rounds where a geriatrician to formulate a management plan. Some patients are reviewed in ad hoc 'Hot Clinics' overseen by geriatricians.

The RRT is comprised of a manager (Band 8c Nurse, manager) , a senior physiotherapist (Band 8b), 16 general nurses, 12 physiotherapists, 3 occupational therapist, 2 para-medics, all trained to be competent in general clinical assessments, a dietitian (part-time), access to a social worker a man for transport and 5 administrators.

Daily ward rounds were undertaken by 2 geriatricians (either EC or LP) with 2 trainees (FY1 and FY2) with the MDT. Week-end ward rounds were undertaken taken by the with the on call geriatrician with the MDT. The service runs from $8 \mathrm{am}$ to $10 \mathrm{pm}$ daily, seven days a week. Out of hours medical support including week-ends was provided by 4 other consultant geriatricians besides EC and LP.

Eligibility criteria are wide. These range from provision of equipment, falls, personal care (washing and dressing); dietitian, managing exacerbations of chronic obstructive airways disease, anticoagulation, non-mobile patients with suspected deep venous thrombosis and patients requiring intravenous therapy e.g.antibiotics for cellulitis or heart failure. STARRS will not manage patients with chest pain, suspected pulmonary embolism, epilepsy, bronchial asthma, suspected gastrointestinal bleed and stroke/Transient Ischemic Attack. Patients were supported in their homes until the medical condition has stabilized or if they need hospital admission. Following that period, care would be transferred to other communitybased services as appropriate.

\section{Study design}

A descriptive evaluation design over a 4 year period was used to monitor service delivery. Data were obtained on patient demographics, source of referrals, response time (time of referral to patients being seen), acceptability of the service, hospital admissions whilst in the service, hospital admissions at 30 days post discharge and a cost evaluation of the service. Quantitative data were obtained from patient case notes and monthly key performing Indices (KPI), 
the later designed jointly developed between the managers of the RRT service and Brent CCG. The KPI for each year starts from April to March the following year. To mitigate the effect of Covid 19, data sets were analyzed from March to Feb for each year up to February 2020. The chi squared test was utilized for statistical comparison.

\section{Results}

\section{Patient's demographics}

Over the past 4 years, 17,626 people were seen by STARRS. The the ratio of females to males was 3:2 with female age from 22 to 107 years and males age between 21 to 104 years. Over $99 \%$ of patients were aged 65 years and above.

\section{Referral (Table I)}

Most of referrals (>70\%) were from General practitioners (GP) with significant increase over the past 4 consecutive years. However, A\&E referrals have significantly declined from March 2018. Likewise LAS referrals have significantly declined over the last year.

Table I Source of referrals

\begin{tabular}{llllll}
\hline Period & $\begin{array}{l}\text { Total number of } \\
\text { referrals I year }\end{array}$ & $\begin{array}{l}\text { \% referrals from general } \\
\text { practitioners }\end{array}$ & $\begin{array}{l}\text { \% Referrals from } \\
\text { A and E }\end{array}$ & $\begin{array}{l}\text { \% referrals } \\
\text { from LAS }\end{array}$ & \% from others \\
\hline March 2016 to Feb 2017 & 3691 & $2561(69) \mathrm{a}$ & $773(21) \mathrm{e}$ & $220(6) \mathrm{i}$ & $137(4)$ \\
March 2017 to Feb 2018 & 4355 & $3093(71) \mathrm{b}$ & $808(18) \mathrm{f}$ & $249(6) \mathrm{j}$ & $205(5)$ \\
March 2018 to Feb 2019 & 4759 & $3457(73) \mathrm{c}$ & $794(19) \mathrm{g}$ & $250(5) \mathrm{k}$ & $158(3)$ \\
March 2019 to Feb 2020 & 4841 & $3623(75) \mathrm{d}$ & $739(15) \mathrm{h}$ & $224(5) \mathrm{l}$ & $525(5)$ \\
\hline
\end{tabular}

Percentages are in parentheses

a vs $b, b$ vs $c$ and $c$ vs $d: p<0.000 I$, e vs $f$ and $g$ vs $h: p<0.00 I$, $f$ vs $g p=0.0002$, i vs $j$ and $k$ vs $I, p, 0.000 I, j$ vs $k p=0.3$

\section{Response times and length of stay (Table 2)}

The two hour response time for patients' clinical encounter have significantly reduced from $82 \%$ in 2016 to $71 \%$ in 2020 at the expense of corresponding increases in patients seen between 2 and 3 hours (from $13 \%$ to $16 \%$ ) and in patients seen after 3 hours (from $5 \%$ to $13 \%)$ over the same period. The mean length of stay in the virtual ward is less than 5 days.

Table 2 Response time from referrals and length of stay

\begin{tabular}{|c|c|c|c|c|c|c|c|}
\hline Period & GP/LAS Total & $\begin{array}{l}\text { Responded } \\
\text { within } 2 \text { hours }\end{array}$ & $\begin{array}{l}\text { Responded within } \\
\text { 2-3hours }\end{array}$ & $\begin{array}{l}\text { Responded } \\
\text { after } 3 \text { hours }\end{array}$ & $\begin{array}{l}\text { Average of LoS } \\
\text { (Days) }\end{array}$ & $\begin{array}{l}\text { Max of LoS } \\
\text { (Days) }\end{array}$ & $\begin{array}{l}\text { Min of LoS } \\
\text { (Days) }\end{array}$ \\
\hline $\begin{array}{l}\text { March } 2016 \text { to } \\
\text { Feb } 2017\end{array}$ & 2791 & $2275(82)$ & $365(13)$ & $|5|(5)$ & 4.9 & 68 & I \\
\hline $\begin{array}{l}\text { March } 2017 \text { to } \\
\text { Feb } 2018\end{array}$ & 3342 & $2581(77)$ & $47 \mid(14)$ & 290 (9) & 4.4 & 47 & I \\
\hline $\begin{array}{l}\text { March } 2018 \text { to } \\
\text { Feb } 2019\end{array}$ & 3707 & $2416(65)$ & $740(20)$ & $550(15)$ & 4.8 & 64 & I \\
\hline $\begin{array}{l}\text { March } 2019 \text { to } \\
\text { Feb } 2020\end{array}$ & 3869 & $274 \mid(7 I)$ & $622(16)$ & $512(13)$ & 4 & 53 & I \\
\hline
\end{tabular}

Percentages in parentheses

Response time (hours) after referrals from GPs or LAS+ A and E referrals were seen the following day after discharge

Others (referrals for iv antibiotics, palliative care, anticoagulation clinic)

\section{Hospital admission avoidance (Table 3)}

HAA from GP and LAS referrals has remained largely unchanged at just over $80 \%$ and under $80 \%$ respectively over the past 4 years. However, HAA from A and $\mathrm{E}$ has consistently declined from $92 \%$ to $87 \%$ over the same period.

\section{Hospital admissions after discharge from RRTat 30 days (Table 4)}

Over the past 4 consecutive years, despite a significant increase in the number of referrals, hospital read mission at 30 days have remained relatively constant at about $10 \%$.

\section{Economic evaluation (Table 5)}

Table 5 illustrates the average tariff for short stay ( 2 days) and 5 days over the past 4 consecutive years with a further economic analysis over the same period based on the number of avoided admissions.

\section{Clinical governance}

Governance was jointly shared between GP and Geriatricians. In additional to monthly KPI, there were regular clinical teaching sessions for STARRS, 3 monthly mortality meetings, audits on service delivery (see some audits in discussion). Satisfaction for the service remain high from both uses and from GPs. Over the last 4 years, there were 9 complaints. 
Table 3 Hospital Admission avoidance (HAA) from different sources and overall hospital admission avoidance whilst under Rapid Response

\begin{tabular}{llllll}
\hline Period & Overall & GP & A and E & LAS & Others \\
\hline March 2016 to Feb 2017 & $3096 / 3691(84)$ & $2090 / 2561(82)$ & $713 / 773(92)$ & $166 / 220(76)$ & $127 / 137(93)$ \\
March 2017 to Feb 2018 & $3720 / 4335(85)$ & $2549 / 3093(82)$ & $778 / 808(96)$ & $194 / 249(78)$ & $199 / 205(97)$ \\
March 2018 to Feb 2019 & $4017 / 4759(84)$ & $2834 / 3457(81)$ & $840 / 894(94)$ & $202 / 250(80)$ & $141 / 158(90)$ \\
March 2019 to Feb 2020 & $4017 / 484 I(83)$ & $2980 / 3623(82)$ & $641 / 739(87)$ & $173 / 224(77)$ & $223 / 255(87)$ \\
\hline
\end{tabular}

Table 4 Overall Hospital admission avoidance at 30 days post discharge from rapid response service

\begin{tabular}{lll}
\hline Peroid & $\begin{array}{l}\text { Overall discharge (admission } \\
\text { avoidance) }\end{array}$ & $\begin{array}{l}\text { Overall admission within } 30 \\
\text { days post discharge }\end{array}$ \\
\hline March 2016 to feb 2017 & 3096 from 369l & 279 (9) a \\
March 2017 to feb 2018 & 3720 from 4335 & $335(9)$ b \\
March 2019-feb 2019 & 4017 from 4759 & $442(1 \mathrm{l}) \mathrm{c}$ \\
March 2019 to feb 2020 & 4017 from 484I & 281 (7) d \\
\hline
\end{tabular}

Percentage in parenthesis, a vs $b \mathrm{p}=0.99$, b vs $c$ and $c$ vs $d, p<0.0001$

Table 5 Average tariff (short stay and 5 day stay) with averaged tariffs based on the number of avoided admissions for the last four consecutive years (20l62020)

\begin{tabular}{|c|c|c|c|c|c|c|c|c|c|}
\hline Period & $\begin{array}{l}\text { Average } \\
\text { short } \\
\text { stay } \\
\text { tarrif }\end{array}$ & $\begin{array}{l}\text { Average } \\
\text { Sday } \\
\text { tariff }\end{array}$ & $\begin{array}{l}\text { No of } \\
\text { patient } \\
\text { avoided for } \\
\text { that year }\end{array}$ & $\begin{array}{l}\text { Total } \\
\text { avoided } \\
\text { tariff- } \\
\text { short stay }\end{array}$ & $\begin{array}{l}\text { Total } \\
\text { avoided } \\
\text { tariff-S day }\end{array}$ & $\begin{array}{l}\text { Costof } \\
\text { STARRS }\end{array}$ & $\begin{array}{l}\text { Saving/ } \\
\text { (loss) } 100 \% \\
\text { short stay }\end{array}$ & $\begin{array}{l}\text { Saving/(loss) } \\
100 \% \text { Sday } \\
\text { stay }\end{array}$ & $\begin{array}{l}\text { Saving/ } \\
\text { (loss) } 50 \% \\
\text { short and } \\
\text { long stay }\end{array}$ \\
\hline & 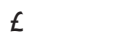 & $£$ & $€$ & $£$ & $£$ & $£$ & $£$ & $£$ & 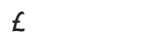 \\
\hline March-16 & 692 & 2319 & 3096 & $21,42,432$ & $71,79,624$ & $-36,87,948$ & $-I, S 45,516$ & $34,91,676$ & $9,73,080$ \\
\hline March-I7 & 824 & 2418 & 3720 & $30,65,280$ & $89,94,960$ & $-37,35,990$ & $-6,70,710$ & $52,58,970$ & $22,94,130$ \\
\hline March-18 & 833 & 2251 & 4017 & $33,46,161$ & $90,42,267$ & $-37,74,564$ & $-4,28,403$ & $52,67,703$ & $24,19,650$ \\
\hline March-19 & 850 & 2518 & 4017 & $34,14,450$ & I,0I,| |4,806 & $-39,34,122$ & $-5,19,672$ & $61,80,684$ & $28,30,506$ \\
\hline
\end{tabular}

\section{Discussion}

With increasing health care costs, an increasing ageing population and greater demand for GP service provision, intermediate care services need expansion and diversification. ${ }^{2}$ Admission avoidance schemes such as hospital at home (with the option of stepping up to acute care) is an attractive alternative for a selected group of older patients requiring acute care. Its role to help resolve crisis, thus avoiding progression and which might otherwise lead to an unscheduled hospital admission. As far as we are aware, this paper has the largest data set to evaluate a home based service to help support the frail older person at home.

Most of referrals (>70\%) were from General Practitioners (GP). This has consistently increased over the past 4 consecutive years. Despite this, admission avoidance was not significantly affected and remained at over $80 \%$ whilst under STARRS. STARRS supported GPs with their increasing clinical work load. GPs were kept up to date daily on their patients either via telephone or emails. Discharged letters are emailed or sent to GP practices within 3 working days after being discharged.

One of the unintended consequences of the increased referrals ( $30 \%$ over the past 4 years) have impacted significantly on the response time. There is a significant reduction in referrals seen within 2 hours and a significant increase in referrals seen between 2 and 3 hours and referral seen greater than 3 hours. Several other factors may also have affected the response time. A large cluster of referrals especially in the late afternoon will significantly stretch the ability of the RRT to see referrals within the time constrains. In some instances, relatives have made specific requests to be present during the assessment. As such, a mutually agreed time would need to be arranged. To help address this, over the past 6 months, subject to the agreement after discussion of each case with individual GPs, referrals are triaged to be seen within 2 or within 4 hours.

However in spite of the increase volume in referrals, the length of stay for patients on the virtual ward remained at less than 5 days. The reduction in the length of stay over the past year may be attributed to that for some patients, they are 'step down' to a similar team to STARRS. There is ongoing support albeit at lower intensity for up to six weeks after the medical crisis has been averted.

HHR30 for STARRS was $80 \%$. This compares favorably with other RRT with avoidance ranging from 75\% to 95\%. ${ }^{3,4}$ However, our data set were from a much larger cohort, over 17,000 compared to Cliff with over 100 patients. Furthermore, some of the patients referred to STARRS may have a higher clinical acuity. STARRS has supported patients with heart failure requiring intravenous diuretics and for non-ambulatory patients requiring intravenous antibiotics. There are increasing numbers of non-medical prescribers and all geriatricians have access to remote electronic prescribing to enable 
prompt initiation of treatment e.g. antibiotics. Data set from Beech et al. ${ }^{4}$ were from a different cohort of 231 patients. The team provided support for patients following hospital admission which may explain a higher hospital avoidance rate at $95 \%$.

Referrals, from A\& E have progressively declined over the last 4 years. Furthermore, HAA from A\& E have also declined over the past 3 consecutive years. Several reasons may account from this. Currently, STARRS do not have "geriatrician presence" at the front door. Patients are assessed by the A \&E team, deemed medically stable before onward referral. A more proactive model such as TREAT may have some impact in same day discharges from A and $E^{5}$ although there were no data on HRA30. In our local hospital "The Frailty Team" with geriatricians at the front door working closely with STARRS in A\&E has just been implemented and are being evaluated. However, the older person presenting to A \& E today may be a frailer cohort compared to in previous years. For those deemed medically stable with onward referral to STARRS, some still elapse and become unwell whilst under STARRS. This may explain a consistent statistical decline in referrals and admission avoidance from A\&E over the last 3 consecutive years.

LAS referrals have also declined in the last year as well as has its associated HAA 30 at less than $80 \%$. Over the past 4 years the number of referrals have remained relatively static at less than 250patients .This figure is only the "tip of an iceberg" compared to the number that LAS receives (Barnes O 2019).STARRS can only accept referrals up to $6 \mathrm{pm}$ daily. LAS referrals to STARRS after this time may have to be conveyed to A \& E. Many patients may be deemed inappropriate for any intervention an initial LAS assessment and therefore were not referred on. A telephone triage service by an LAS "clinician" to replace the face to face assessment is currently under discussion, to divert referrals directly to STARRS. However, there needs to be a robust mechanism for this telephone triage service with medical accountability so as not to compromise the frail older person. Some may require an emergency service of which STARRS is not.

A HRA30 at $10 \%$ is much lower compared to hospital admission rate at $15 \%$ from patients discharged from the care of the elderly wards. ${ }^{6}$ This may suggest that, overall, patients have a lower clinical acuity. Indeed this should be the "norm" as STARRS should not be delaying hospital admissions for the older person who needs it. Hospital admission rate at 90 days (HRA90) was previously included as part of the KPI for the service. From April to Dec 2015 the average rate was at $14.7 \%$. A more recent audit on 378 consecutive patients for the month of Jan 2109 was $14.8 \%$ (Audit Mohammad K and Shah K 2020).

One criticism of any RRT is the potential to extend the length of hospital stay if patients are admitted, possibly increasing morbidity and mortality during the hospital admission. This is very difficult to substantiate or refute this.

In Jan 2014, we reviewed the outcome on 151 consecutive hospital admissions over a 6 month period after being accessed by STARRS (Audit: Levy, Potter and Kennard 2015).Twenty four passed away whilst in hospital. Of these, 14patients were admitted within Day 2 from referrals. Ten were admitted after day 2 (range 3 to 7 days). Two out of 10 passed away within 3 days of hospital admission (1 patient declined hospital admission on Day 2 but eventually come in on Day 5 , the other patient was a discharged from A\&E to her residential home, but was re-admitted on Day 5 post discharge). The remaining 8 patients stayed in hospital for greater than 10 days before they passed away.
In Jan 2018 we audited the length of stay (LOS) on Day 1 admission by STARRS for 1 year (AuditKolliV 2018) This revealed wide variations in LOS. From 170 patients, LOS was available for 111 patients, 59 went to alternate hospitals. Of 111, $11(10 \%)$ were discharged within 2 days, $12(11 \%)$ had a length of stay under 5 days, $43(39 \%)$ stayed in hospital for up to 10 days and $45(40 \%)$ stayed for greater than 10 days (99 days for one patient).

Unplanned hospital admissions (emergency admissions) have increased by nearly $50 \%$ in the past 15 years (3.6 million in 1997 to 5.3 million in 2012). Emergency admissions now account for up to $67 \%$ of all hospital bed days in England. ${ }^{7}$ Several factors have been cited to explain this; an ageing population, introduction of financial incentives (abolition of block contracts), yearly decline in social care funding and new performance targets for health care providers. RRT's are becoming increasingly common in different regions of the United Kingdom, However, A \& E departments remained busy except more recently, with Covid 19 with reduced attendance by $50 \%$.

In 2008, it is estimated that over 80 million people in Europe will be over 65 years old of which nearly $25 \%$ will be over the age of 80 years old (old old). In this year the over 65 year old group will increase by $21 \%$ ( 18.4 million in real terms) with the old-old at a greater increase by $34 \%$ (additional 7.5 million). ${ }^{8}$ Over the past 2 decades, health care have achieved better outcomes e.g. Stroke Units, ${ }^{9}$ the management of acute coronary syndromes ${ }^{10}$ orthogeriatic liaison services $^{11,12}$ and proactive surgical interventions and medical support post- surgery for the older person. ${ }^{13,14}$ This have created an increasing frail cohort of patients, who upon discharge from hospital have a high risk for subsequent hospital re-attendances. ${ }^{15}$

Lauicella ${ }^{16}$ examined several patient cohorts between April 2000 and March 2010. They labelled a patient's first admission as the index admission and followed patients in each cohort for 2 years after discharge and for 2 years before from their index admission. Patients with any history of emergency admissions within 2 years before the index admission were excluded. This was to avoid confounding effects from the care received during past admissions.

Patient cohorts include post hip fracture (65years and above), acute myocardial infarction (AMI) aged55 and above, strokes aged 55 and above and all-causes for emergency admissions aged 55 and above. In total, there were over 286,000 patients in the hip fracture cohort, 375,000 in the AMI cohort, 387,000 in the stroke cohort and just under 10 million with any cause emergency admission.

Their analysis of for all-cause emergency admissions revealed improved survival rates by an additional 4.07 patients per 100 admissions between 2000 and 2009. This resulted in an additional 7.73 subsequent emergency admissions per 100 admissions in 2009 . When they rescaled this to their study population there will be an additional 1millon patients emergency admission in 2009. This equates to an additional 84,000subsequent admissions within 1 year of discharge due to better survival rates. For the period 2000 to 2009 , they calculated this to be an additional $37.3 \%$ increment in emergency admissions (an additional nearly 226,000attendances). Similar analysis can be undertaken for hip fractures, AMI and stroke.

Furthermore, a sizable proportion of this cohort will become the "frequent user of medical services". This phenomenon is well known to medical practitioners involved in the management of chronic illness. Similarly for STARRS, in a retrospective audit on 391 consecutive patients referred for the month of December 2017,75\% had up to 2 contacts, $15 \%$ had 4 and $10 \%$ had greater than 5 contacts with STARRS in the previous year (Audit Azeliaand Sharma 2019). 
Similarly, in aprospective study on321consecutivepatients(mean age 84 years old) discharged from our inpatient intermediate care rehabilitation unit between January to December 2016, at 1 year post discharge, 174 patients (55\%) needed hospital services; of which 129 patients $(73 \%)$ were within 3 months post discharge. Within the 3 month cohort, 38 patients $(30 \%)$ had 3 or more hospital encounters (range 3 to 8 ) and $34(11 \%)$ were dead within a year. (Audit Risma Pandya R and Chua P 2017).Management of this cohort remains challenging as effective evidenced based models of care remain elusive.

Response teams similar RRT, with lower intensity but with the flexibility to manage over a more prolonged period for up to several weeks has emerged. Operating in the same principles as the RRT, some patients can be "step down" from STARRS after cessation of a crisis or, for patients after a prolonged hospital stay. Efficacy of such a model of care is currently being evaluated.

We also attempted to undertake an economic analysis of the RRT .We are aware that there several constraints when one undertakes this task. Medical opinions can differ on what constitutes a genuine hospital avoidance from clinician to clinician.(Audit ChuaE and Patel D 2016). Furthermore, when patients are admitted, it is difficult to ascertain their length of stay in hospital due to variations between patients. As mentioned, in our previous auditon 111 consecutive patients admitted on Day 1, the duration of stay varied from 2 to 99days (Audit V Kolli, M Mistry and E Chua 2018).

For these reasons we undertook this analysis based on the average tariff for short stay (2 days) and mean duration of stay of 5 days. As illustrated in Table 5, for the last financial year, the average short day tariff suggests that the service might be in negative equity by half a million whereas, the 5 day tariff, puts the service at a positive equity at over 6 million pounds. We believe the figure is likely to be in between the two. If this was indeed the case, it would appear that the Rapid response service may be economically viable with potential savings in addition to benefits of maintaining the frail older personin their homes. However, it needs to be emphasized that the potential gains to the older person in avoiding a hospital admission must not be undertaken at the cost of a missed or delayed diagnosis. Therefore, Primary Care Physicians should be reviewing patients before onward referral.

\section{Conclusion}

The NHS and social care system are constantly under great strain with an ever increasing demand. In the $21^{\text {st }}$ century, medicine has succeeded in stabilizing medical conditions on an increasing number of patients. However, they remain frail and therefore at high risk of accessing future emergency care. Targeting health resources for these persons will bekey to help reduce demand for subsequent emergency care. RRT may be one of a range of services to help support this.

\section{Audits were performed by the following}

Mohammad K and Shah K (2020) 90 day hospital admissions on 378 consecutive patients post discharged in January 2019 compared to 2906 patients from April 2015 to Dec 2015.

Levy S, Potter A and Kennard D (2015): Mortality Audit on 152 consecutive patients admitted whilst under STARRS (Nov 2014 to April 2015)
RushdA and Prasad N (2019): Retrospective audit 391 consecutive patients (Dec 2018 to Jan 2019)seen by STARRS in the previous year.

Chua E and Patel D (2016) Review of 22 patients with "disputed" hospital avoidance.

Kolli S, MistryM and Chua E (2018) : Audit on 111 consecutive admission and length of hospital stay following Day 1 admission by STARRS.

Pandya R and ChuaP (2017) Prospective audit on 321 consecutive patients (Jan 2016 to Dec 2016) and hospital attendance a year following discharge.

Armstrong J, Head of Finance, London North-West University HealthCare NHS Trust for her work in the costings.

\section{Acknowledgments}

Barnes O, North-West London Clinical Commissioners Group, Oct 2019, Data set on Brent LAS referrals for period January 2019 to July 2019

\section{Conflicts of interest}

The authors declare have no conflict of interest about the publication of this paper.

\section{References}

1. Brent demographics: UK National Statistics. 2018.

2. Department of health The NHS plan: a plan for investment, plan for reform. London HMSO. 2000.

3. Cliff, E. How a rapid response team is supporting people to remain a home. Nursing older people. 2015:(10)16:18-21.

4. Beech R, Russell W, Little R, et al. An evaluation of a multidisplinary team for intermediate care at home. Int J Integr Care. 2004.

5. Wright $\mathrm{P}$, Tan $\mathrm{G}$, Iliffe $\mathrm{S}$, et al. The impact of a new emergency admission avoidance system for the older person on the length of stay and same day discharges. Age Aging. 2013; 43(1):116-121.

6. Zerdevas PDC. Emergency readmission rates:, further analysis. London Department of Health. 2008

7. http://www.nao.org.uk/report/emergency-admissions-hospitalsmanaging-demand

8. Eurostat. Ageing characteristics the demographic perspectives of the European societies. Luxembourg, for official publications of the European Communities. 2008.

9. Langhorne P1, Williams BO, Gilchrist W, et al. Do stroke units save lives?. Lancet. 1993:14;342(8868):395-398.

10. Déganoa R, Subiranac I, Danilo FB, et al. on behalf of the EUROTRACS investigators.Percutaneous coronary intervention reduces mortality in myocardialinfarction patients with comorbidities: Implications for elderly patients with diabetes or kidney disease. International of Cardiology. 2017:249;83-89.

11. Middleton M. Orthogeriatrics and hip fracture care in the UK: factors driving change to more integrated models of care. Geriatrics. 2018;3(3):55.

12. Konstantin V. Grigoryan, MS, Houman Javedan MD, et al. Ortho-geriatric care models and outcomes in hip fracture patients: a systematic review and meta-analysis. J Orthop Trauma. 2014;28(3):e49-e55. 
13. Wilkinson K. An age old problem: a review of the care received by elderly patients undergoing surgery: a report by the national confidential enquiry into patient outcome and death. London National Confidential Enquiry into Patient Outcome and Death. 2010.

14. Partridge JS, Harari D, Martin FC, et al. The impact of pre-operative comprehensive geriatric assessment on postoperative outcomes in older patients undergoing scheduled surgery: a systematic review. Anaesthesia. 2014;69:8-16.
15. Laudicella, M, Li Donni P, Smith PC. Hospital readmission rates: signal of failure or success?. Journal of Health Economics. 2013;32 (5):909-921.

16. Laudicella M, Martin S, Li Donni P, et al. Do reduced hospital mortality rates lead to increased utilization of inpatient emergency care? a populationbased cohort study. Health Service research. 2017;53(4):2324-2345. 